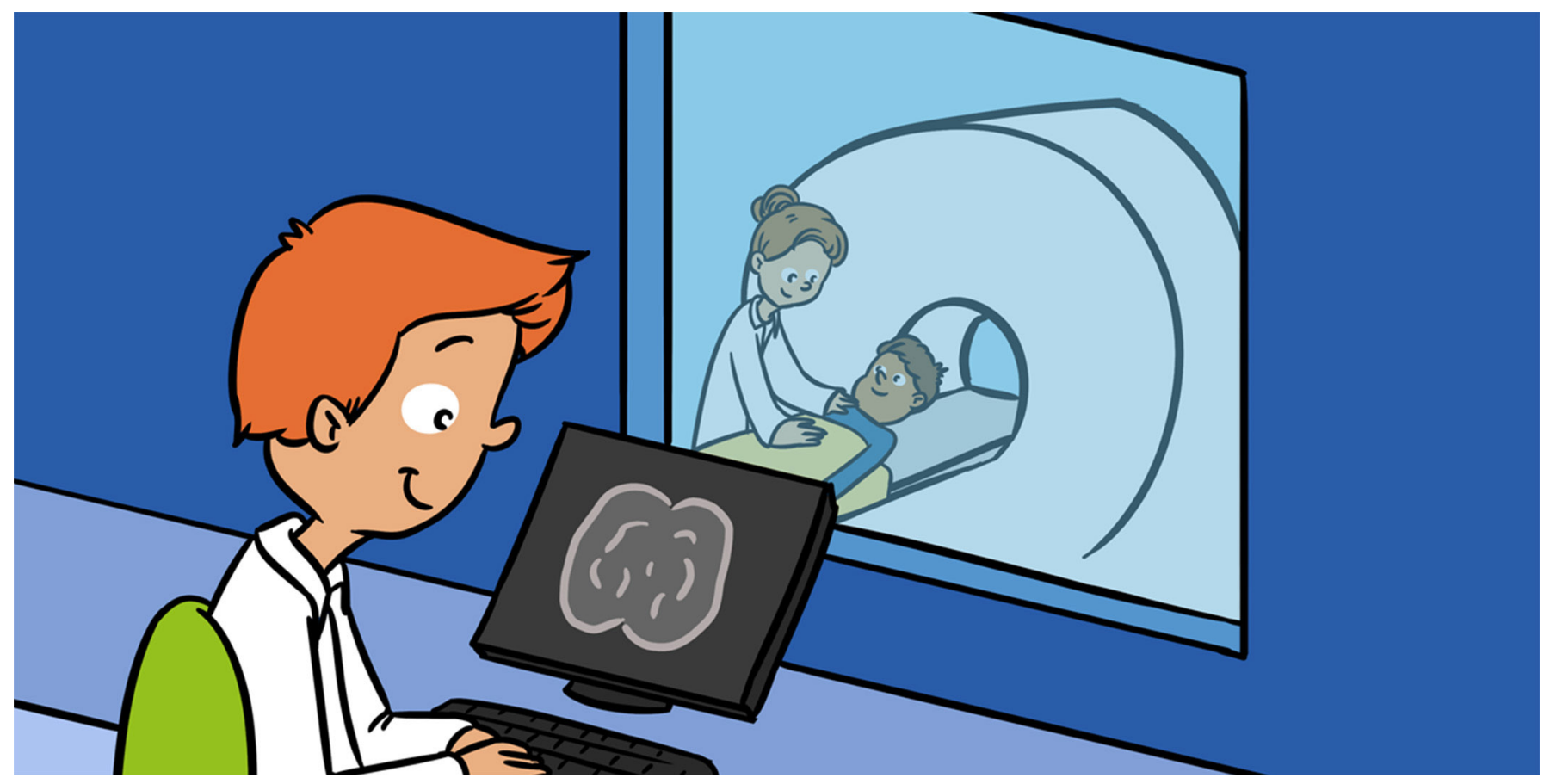

\title{
HOW TO SEE INTO THE BRAIN WITHOUT SURGERY!
}

\section{Christine L. Mac Donald ${ }^{1 *}$, Pratik Mukherjee ${ }^{2}$ and Esther Yuh ${ }^{2}$}

${ }^{1}$ Department of Neurological Surgery, University of Washington, Seattle, WA, United States

${ }^{2}$ Department of Radiology, University of California, San Francisco, San Francisco, CA, United States

YOUNG REVIEWERS:

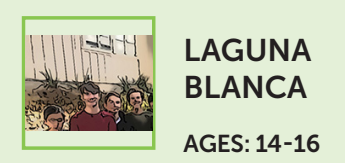

Did you know that doctors look at the brains of thousands of people every day? In hospitals all over the nation, we are looking into patients' brains to see if something has gone wrong, so we can understand how to help treat each patient's condition. Brain imaging technology plays an important role in helping doctors diagnose and treat conditions like brain injuries. Behind the scenes, there are special cameras that allow us to see deep inside of the brains of patients every day.

\section{INTRODUCTION}

The field of medicine that is focused on capturing and analyzing images of the brain and all parts of the body is called radiology. The name radiology comes from the first type of "camera" or technology that was used to take these pictures, called the X-ray machine, which uses radiation. The word radiology was derived from the word parts "radio," for radiation, and "ology," from the Greek suffix meaning the scientific study of something. The invention of the X-ray is credited to German 
Figure 1

An X-ray of the hand of Anna Ludwig taken on 22 December 1895 by her husband Wilhelm Röntgen. This is thought to be the first medical X-ray (Image credit: Otto Glasser, Wilhelm Conrad Röntgen and the early history of the Roentgen rays. London, 1933. National Library of Medicine)
CT

Computed tomography.

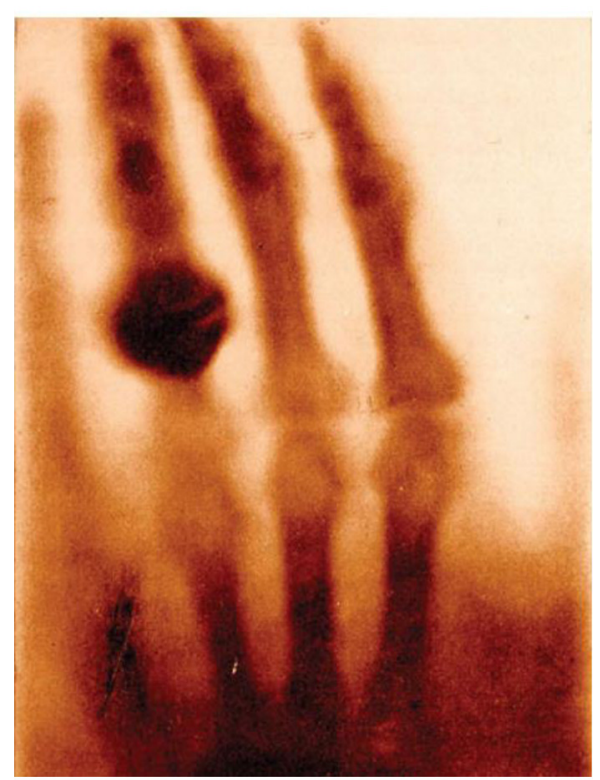

Figure 1

mechanical engineer Wilhelm Conrad Röntgen who, in 1895, generated and detected the electromagnetic radiation that became the basis of the X-ray technology we still use today. In Figure 1, you can see the bones of Röntgen's wife's hand, thought to be the first X-ray image of a human body part. You can see the different finger bones, called phalanges, and a dark blob on the ring finger, which is her wedding ring. If you have ever broken a bone, you likely had an X-ray to determine where the break occurred.

\section{HOW DO DOCTORS LOOK INSIDE THE BRAIN?}

When a patient has a brain injury, one of the first things radiologists do in the emergency department of the hospital is to use a special kind of "camera," called a computed tomography (CT) scanner, to look at the patient's brain. The CT scanner, as it is known for short, is a machine that allows doctors to see into a patient's brain without surgery. The CT technology uses small bursts of radiation that pass through the body, similar to X-rays, and create different signals depending on the type of tissue they pass through, be it skin, bone, brain, or other tissue types. All of these signals are collected by a computer, which reconstructs them to form a three-dimensional image of the brain that the radiologist can analyze on a computer to look for injuries. The main difference between $\mathrm{CT}$ and $\mathrm{X}$-ray is that $\mathrm{X}$-ray just creates a single, two-dimensional image, similar to a picture you would take with your cell phone. While both the X-ray and CT can collect images in a matter of seconds, both techniques use radiation, and repeated radiation exposure can increase one's risk of various cancers. Radiology has even 
Figure 2

A patient being placed into a magnetic resonance imaging (MRI) scanner.

\section{MRI}

Magnetic resonance imaging.

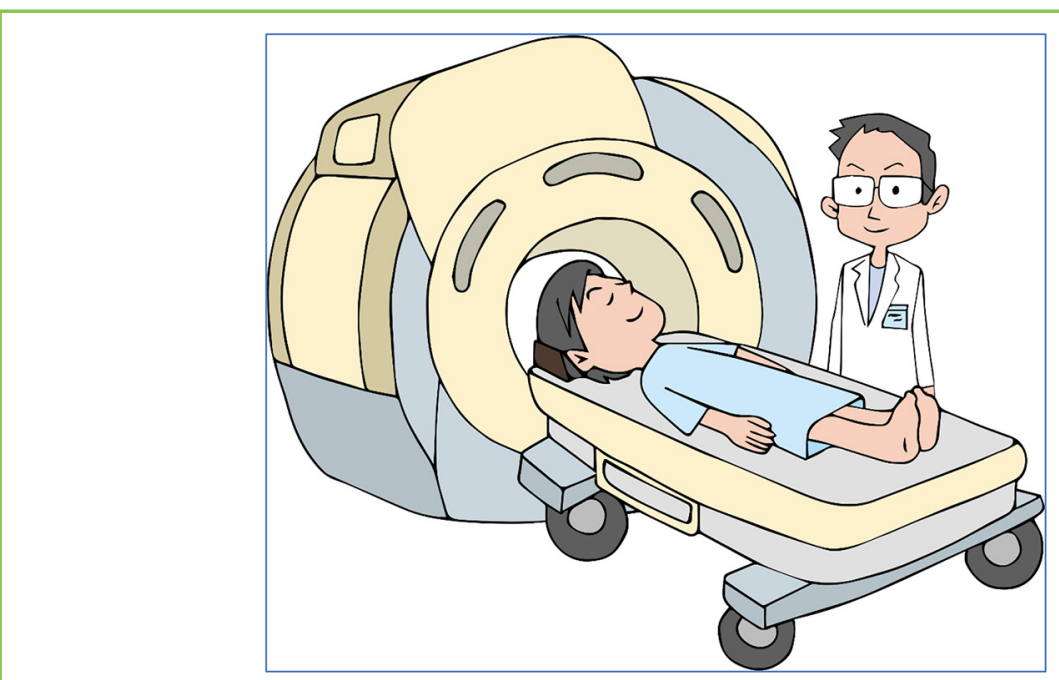

Figure 2

gone so far as to engineer what are called "low dose" CT scanners, used most often at children's hospitals, to try to reduce the amount of radiation young patients are exposed to.

In addition to CT scans, another imaging technology called magnetic resonance imaging (MRI) can also be used to look inside the brain. MRI scanners use very strong magnets to look at the brain by using the "excitability" of the water molecules in our bodies. When we are young, our bodies are made up of $70-80 \%$ water and as we age, that proportion goes down to $50-60 \%$, so there is a lot of water in our bodies to help with this kind of imaging! The magnets that MRI scanners use are measured in a unit called Tesla (T). These are very strong magnets, much stronger than the earth's magnetic field, which measures between 25 and 65 microTesla ( $\mu T$, one millionth of a Tesla). The magnetic strength of clinical MRI scanners is 1.5-3 Tesla, 30,000-60,000 times stronger than the earth's magnetic pull! Because of these strong magnets, all patients are carefully screened for metal or implantable electronic devices, like cardiac pace makers, before they are put into an MRI scanner because the magnets could excite these materials and potentially cause harm to the patient. To date, no safety concerns have been identified with repeat MRI, as long as the patient does not have any MRI-incompatible devices or materials in his or her body.

For brain imaging with $\mathrm{CT}$ and $\mathrm{MRI}$, the patients are most often asked to lie on their backs and then they are placed in the scanner, which is built like a tube. When imaging the brain, the patient's head is placed in the center of the tube where the machine has the best, most uniform, imaging strength (Figure 2). 


\section{COMMON BRAIN INJURIES THAT NEED IMAGING}

Following a traumatic brain injury, there are various kinds of damage to the brain that imaging technologies can help doctors to see. Good imaging methods that let doctors see the entire brain are very important, in order to figure out exactly how the brain has been injured. The most common injuries are small bleeds in the brain, called microbleeds or microhemorrhages (see Figure 3), meaning a small ("micro") escape of blood from a torn blood vessel ("hemorrhage"). If you get a nosebleed, this is also a form of microhemorrhage-of the nasal membrane. The brain needs a continuous supply of oxygen from the blood to perform its everyday functions. The oxygen travels to the brain via blood vessels that come in a variety of sizes, from large arteries down to tiny capillaries. The smaller vessels are thought to be particularly vulnerable to brain injury, because they can stretch and compress as the brain gets jostled around in the skull during an injury. On a CT scan, these microhemorrhages look different from the surrounding brain tissue and have a clear "signature" as the blood pools in the area (see arrows pointing to dark dots in the left panel of Figure 3). MRI scanning is a more sensitive way to identify these microhemorrhages and other types of brain injury [1], but MRI takes longer than CT, so when there is severe trauma, CT is still more commonly used to get a quick image of what is going on in the brain. Another strength of MRI over CT is that it can be used to take different kinds of pictures, whereas the CT only takes one type of picture. With $\mathrm{MRI}$, we can control the magnetic excitation properties of the molecules in ways that change the contrast on the images we collect. Where one type of MRI image may make blood show up brightly, another may make it show up dark, and these differences in contrast help radiologists more confidently determine that they are seeing something abnormal in the brain, like blood, or bruises to the brain tissue from an injury.

Another common brain injury is called a contusion (see arrows pointing to the bright spot in the lower left of the center panel in Figure 3), which is a bruise on the brain due to trauma. Like bruises that you get on your arm or leg from a fall, a brain contusion often includes a collection of

Figure 3

MRI images of brain injuries. Left to Right: microhemorrhage, contusion, subdural hematoma.
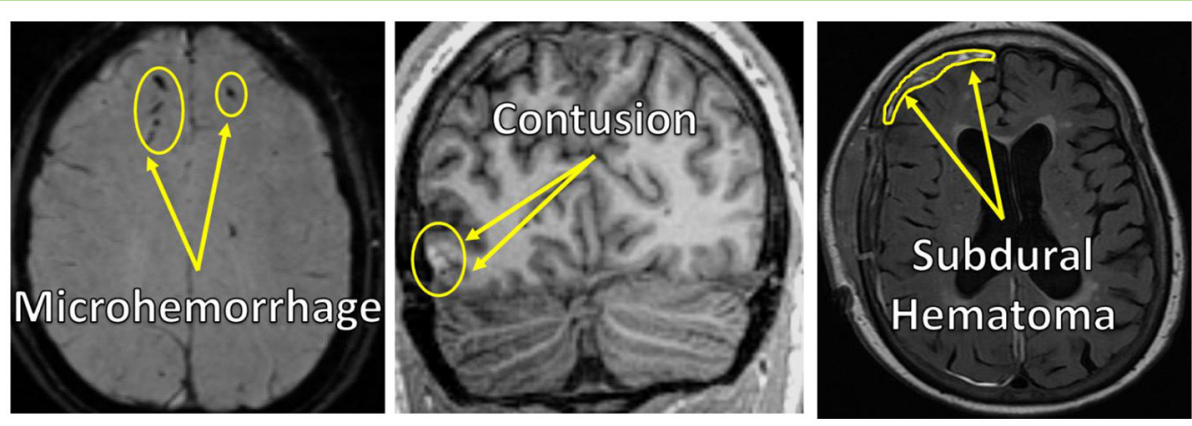

Figure 3 
bleeds in a localized area. Unlike a bruise on your arm or leg, which heals over time, leaving no mark of the injury, brain contusions and the damage to the neighboring brain tissue can be more permanent. Looking inside the brain using CT or MRI imaging allows doctors to visualize brain contusions, which helps doctors determine how best to diagnose, treat, and care for patients with traumatic brain injury.

In addition to microhemorrhages and contusions, there are a variety of traumatic brain injuries that occur in what is called the "extra-axial space." The extra-axial space is the space between the surface of the brain and the inside of the skull. There are different compartments of the extra-axial space, called the subdural (see arrows pointing to the bright band in the upper left of the right panel in Figure 3), epidural, or subarachnoid extra-axial spaces. These compartments consist of different layers between the brain and the skull and they contain a variety of blood vessels that can break open, causing a pooling of blood termed hematoma. Hematomas most often occur with more severe head injuries that happen at a greater force and acceleration.

Lastly, just like with an ankle sprain, the brain can also swell following an injury or can contain a region of pooled fluid, which is called "edema." Doctors rely heavily on CT and MRI imaging to monitor the brain for edema because, unlike a sprained ankle where the skin can expand a bit as swelling occurs, the skull is a very stiff bony structure that does not expand when the brain swells, which may cause additional damage if the brain swelling is not treated quickly.

\section{CONCLUSION}

Advances in technology play an important role in the ability of doctors to diagnose and treat head injuries in a team approach. From emergency medicine physicians who make the initial evaluations, to radiologists who review and analyze the brain images, to neurosurgeons who may operate on a patient's brain, there is a diverse team of doctors who work together to provide the best treatment possible to each patient. And behind the scenes, there are these "special cameras," CT and MRI that allow them to see inside the brains of thousands of patients every day.

\section{REFERENCE}

1. Yuh, E. L., Mukherjee, P., Lingsma, H. F., Yue, J. K., Ferguson, A. R., Gordon, W. A., et al., TRACK-TBI Investigators. 2013. Magnetic resonance imaging improves 3 -month outcome prediction in mild traumatic brain injury. Ann. Neurol. 73:224-35. doi: 10.1002/ana.23783 
SUBMITTED: 24 September 2018; ACCEPTED: 21 January 2019;

PUBLISHED ONLINE: 08 February 2019.

EDITED BY: Amy J. Markowitz, University of California, San Francisco, United States

CITATION: Mac Donald CL, Mukherjee P and Yuh E (2019) How to See Into the Brain Without Surgery! Front. Young Minds 7:14. doi: 10.3389/frym.2019.00014

CONFLICT OF INTEREST STATEMENT: The authors declare that the research was conducted in the absence of any commercial or financial relationships that could be construed as a potential conflict of interest.

COPYRIGHT @ 2019 Mac Donald, Mukherjee and Yuh. This is an open-access article distributed under the terms of the Creative Commons Attribution License (CC BY). The use, distribution or reproduction in other forums is permitted, provided the original author(s) and the copyright owner(s) are credited and that the original publication in this journal is cited, in accordance with accepted academic practice. No use, distribution or reproduction is permitted which does not comply with these terms.

\section{YOUNG REVIEWERS}

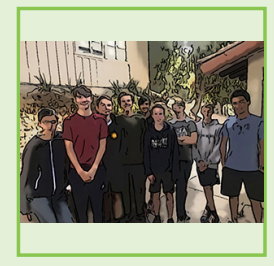

\section{LAGUNA BLANCA, AGES: 14-16}

Students enrolled in their first year of a 2-years science research program. Everyone in class has a different area of interest, so no matter what authors submitting to Frontiers of Young Minds write about, we would always be up for offering our input.
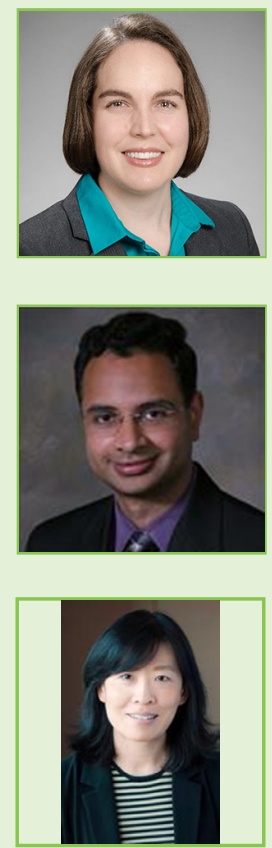

\section{AUTHORS}

\section{CHRISTINE L. MAC DONALD}

I am an Associate Professor and the James and Gaye Piggott Endowed Chair of Neurological Surgery at the University of Washington (UW) School of Medicine in Seattle, Washington. I am also the Research Director of the Sports Institute at UW Medicine. ${ }^{*}$ cmacd@auw.edu

\section{PRATIK MUKHERJEE}

I am a Professor of Radiology and a board-certified Neuroradiologist at the University of California San Francisco in San Francisco, California. I am also the Director of the Center for Imaging of Neurodegenerative Disease (CIND) based at the San Francisco VA Medical Center.

\section{ESTHER YUH}

I am an Associate Professor in Radiology and a board-certified Neuroradiologist at the University of California San Francisco in San Francisco, California. I lead major efforts in the neuroradiological evaluation of traumatic brain injury in collaboration with Drs. Mac Donald and Mukherjee. 